\title{
A Scientific Comparison of Three Tests for Swallowing Dysfunction That Are Not Reliant on Access to VF or FEES Facilities
}

\author{
Mary Hägg*1,2 and Lita Tibbling ${ }^{3}$ \\ ${ }^{1}$ Speech \& Swallowing Centre, Department of Otorhinolaryngology, Hudiksvall Hospital, Sweden \\ ${ }^{2}$ Research \& Development Centre, Uppsala University, Sweden
}

${ }^{3}$ Department of Otorhinolaryngology, Linköping University, Sweden

*Corresponding author: Mary Hägg, DDS, PhD, Speech \& Swallowing Centre, Dep. of Otorhinolaryngology, Sweden

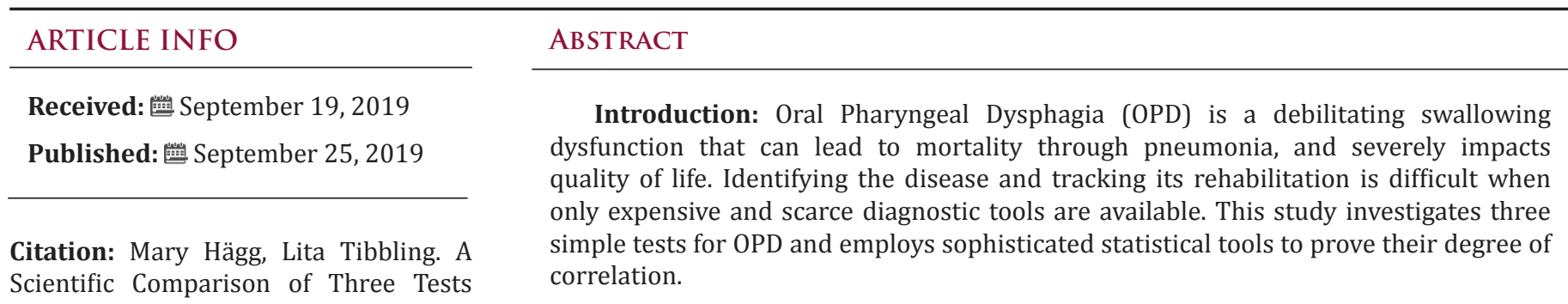
Not Reliant on Access to VF or FEES

Objectives: To compare the Timed Water Swallow Test (TWST) with a Meal Observation Test (MOT) and Visual Analogue Scale (VAS) as tools for detecting OPD and its treatment.

Methods: Non-hospitalized stroke patients $(\mathrm{n}=22)$ with OPD (median 15.5 months after stroke) were assessed using TWST (ml/s), a 5-item MOT (score 0-4), and a VAS (0$100 \mathrm{~mm}$ ) for swallowing difficulties: at baseline, at 3 month end-of treatment and at long term follow-up -averagely 14 months post treatment.

Results: All tests correctly identified almost all patients as having pathological levels for OPD at baseline. There was very good correlation of the three tests' results at baseline, end-of-treatment and at long term follow-up.

Conclusion: It seems that TWST has the widest degree of scientific evidence, and that both MOT and VAS give similar levels of certainty in diagnosing OPD. The lack of correlation of VAS with the other test methodologies suggests that caution is needed when deploying this method alone in patient evaluation; this may because of the hypothesised phenomenon of the patient's observation baseline changing over time. This study demonstrates powerful alternatives to the clinician when preferred, or when more specialist VF or FEES resources are not readily available.

\section{Introduction}

Oropharyngeal dysphagia (OPD) is a common cause of bronchopulmonary complications due to aspiration [1,2]. OPD can also cause malnutrition and a psychosocial handicap with impaired quality of life, especially in elderly patients [3-5]. Recently, neuromuscular training has had promising treatment results in stroke patients with long-standing OPD [6,7]. To allow health professionals and caring staff to detect and diagnose OPD a simple test is needed that can confirm the presence of the swallowing dysfunction, and to assess the effect after rehabilitation treatment.
In well-equipped hospitals there is often access to video fluoroscopy or FEES facilities, although these are still precious resources that are not always accessible. In intermediate care or residential care homes, such equipment is available only by referral to hospital. The aim of this study was to investigate the reliability of three different patient swallowing tests that can be performed without recourse to expensive, scarce or invasive examination methods. Patients' swallowing dysfunction was measured by the three different tests at baseline, end-of-treatment and at long term follow-up, post 
treatment. The results of each methodology were compared at all three timepoints, and the results analysed for correlation.

\section{Material and Methods}

\section{Study Population}

The study included 22 patients: 7 women and 15 men, median age 69 years (range 47-82) with long-standing OPD as assessed by the referring physicians. Other inclusion criteria were first-ever stroke, no other central neurological diseases, and the ability to cooperate. A total of 48 patients were referred, 26 of whom could not complete the study: 18 mortalities, 5 new stroke, and 3 unable to cooperate or tube-fed. The baseline study started at median 15.5 months (range 1-108) after stroke and was followed by a 3-month training period. A long-term follow-up was performed on average 13.5 months (range 4-62) after end-of-treatment. The study period ran from 2007 to 2014 .

\section{The three test methodologies used}

Timed Water Swallow Test (TWST): The patient was placed in a sitting position - to reduce the risk of misdirected swallowing - and initially given a teaspoon of water to swallow 1-3 times. If the patient was unable to swallow anything at all, or immediately experienced misdirected swallowing, the TWST was scored as zero. Otherwise, the patient was instructed to swallow $150 \mathrm{ml}$ of water from a glass as quickly as possible without pausing, but safely, and to stop if any difficulty arises. The time from start of drinking until completion of the final swallow was recorded. The water remaining in the glass, if any, was measured. Swallowing rate $(\mathrm{ml} / \mathrm{s})$ was defined as the amount of water swallowed divided by time. A swallowing rate of $10 \mathrm{ml} / \mathrm{s}$ is regarded as the lower normal limit $[8,9]$. The TWST has been previously shown to have high intrarater, inter-rater, and test-retest reliability $[8,9]$. The TWST has been shown to be a reliable and valid method for assessing swallowing rate in neurological patients with disordered swallowing and thus to be valuable for monitoring therapeutic improvements $[8,9]$.

Meal Observation Test (MOT): A test meal consisting of $2 \mathrm{dl}$ yogurt with jam, one piece of crispbread with butter, and $1.5 \mathrm{dl}$ of fruit squash was served to each patient. The patient sat upright in a chair and five observation variables were scored from 0 (normal) to 4 (severe dysfunction) by two separate investigators.

i. Oral transit time: Oral preparation time from intake to excitation of the swallowing reflex (larynx elevation): $0=<10$ sec, $1=$ sometimes $>10 \mathrm{sec}, 2=$ often $>10 \mathrm{sec}, 3=$ always $>10$ sec, $4=$ complete inability to swallow.

ii. Drooling: $0=$ never, 1 = sometimes, 2 = often, $3=$ constantly, $4=$ complete inability to keep saliva and food in the mouth.

iii. Cough: $0=$ never, $1=$ seldom, $2=$ sometimes, $3=$ often, $4=$ always.

iv. Hoarseness: If hoarseness was observed after the meal, the patient was asked how often this symptom was experienced: 0
= never, 1 = seldom, 2 = sometimes, 3 = often, 4 = always.

v. Nasal leakage: If nasal leakage was observed after the meal, the patient was asked how often this symptom was experienced: $0=$ never, $1=$ seldom, $2=$ sometimes, $3=$ often, 4 $=$ constantly.

The MOT score for each patient was recorded as the mean of the sum of all five item scores giving an overall:

$0=$ normal, $1=$ almost normal, $2=$ moderate impairment, $3=$ severe impairment, $4=$ total inability.

The intra-rater and inter-rater reliability of the MOT has previously been shown to have a high kappa coefficient (1.00) [10].

\section{Visual Analogue Scale (VAS)}

In this test, we ask each patient to rate their own perception of their swallowing difficulties in daily life. They indicate on a 0 $100 \mathrm{~mm}$ VAS ruler how they rate that their swallowing affects their mealtimes. Their results are rated as follows:

$0=$ normal, $1-20=$ almost normal, $21-99=$ pathological; $100=$ total inability.

These were assessed on three different occasions: at baseline, at end-of-treatment at 3-months, and at long term follow-up averagely 14 months post treatment.

\section{Training Intervention}

The patients $(n=22)$ all trained for 3 months; some $(n=13)$ underwent oral neuromuscular training $1 \frac{1}{2} 2$ minutes per day with an IQoro ${ }^{\circledR}$ device (Figures $1 \mathrm{a} \& 2$ ), and the rest $(\mathrm{n}=9)$ for $1 \frac{1}{2}$ hours daily, with a palatal plate (Figure $1 \mathrm{~b}$ ).

When training with IQoro, the device is placed in the mouth pre-dentally behind closed lips and the patient pulls forwards on its handle whilst retaining the IQoro with the lips. Three daily exercise sessions were performed: each consisting of three pulls of 5-10 seconds duration.

The palatal plate exercise [10] required the plate to be inserted in the upper jaw for three separate exercise sessions during the day. Each of these exercise sessions was of 30 minutes' duration. The patient was asked to exercise by touching various components of the plate with the tongue according to the given instructions."

\section{Statistical Analysis}

Professional statisticians and a data manager from the Uppsala Clinical Research Center were involved from the outset in planning the study design in accordance with Good Clinical Practice (GCP). They constructed a database to consolidate and analyse all collected data according to the initial protocol. Wilcoxon's test was used to analyse changes in each MOT item between the three different test occasions. Spearman correlation was used to assess agreement between how TWST, MOT, and VAS classified each item: whether as normal or pathological. The following scores were deemed 
pathological: TWST $<10 \mathrm{ml} / \mathrm{s}$, MOT points $>1$, and VAS $>20 \mathrm{~mm}$; and a $\mathrm{p}<0.05$ was considered significant. All statistical analyses were performed using SAS version 9.1 (SAS Institute Inc., Carey, NC, USA).

\section{Results}

\section{Results Per Test Methodology}

a) TWST: At baseline, only 2 of the 22 patients in the study exhibited normal scores for swallowing rate. At endof-treatment this had improved to 14 (64\%) patients having achieved normal swallowing rate levels. By long term follow- up 13 exhibited a normal rate; the majority had continued to improve their rate of swallow (Figure 3 and Table 4).

b) MOT: At baseline, no patients showed a normal score, all had pathological test levels with a mean score of 2.0 (range 1.2 - 3.0). Coughing was observed in $100 \%$ of patients, drooling in $91 \%$, hoarseness in $86 \%$, prolonged oral transit time in $82 \%$, and nasal leakage in 59\% (Table $1 \& 4$ ). All patients exhibiting hoarseness after a meal also experienced coughing during the meal. At end-of-training 13 (59\%) exhibited a normal MOT score. The mean MOT score for the whole group improved significantly $(0.8$, range $0-1.6, \mathrm{p}<0.0001)$.

Table 1: Meal Observation Test Items.

\begin{tabular}{|c|c|c|c|c|c|}
\hline \multirow{2}{*}{ Item } & Baseline & End & Follow-up & $\mathbf{B}-\mathbf{E}$ & $\mathbf{E}-\mathbf{F}$ \\
\hline & $n=22$ & $n=20$ & $n=22$ & p-value & p-value \\
\hline \multirow{2}{*}{ Oral transit time } & $18(2.2)$ & $14(1.1)$ & $6(1.0)$ & \multirow{2}{*}{$<0.001$} & \multirow{2}{*}{$<0.02$} \\
\hline & $18 \%$ normal & $30 \%$ normal & $73 \%$ normal & & \\
\hline \multirow{2}{*}{ Drooling } & $20(2.6)$ & $15(1.0)$ & $7(1.0)$ & \multirow{2}{*}{$<0.001$} & \multirow{2}{*}{$<0.02$} \\
\hline & 9\% normal & $25 \%$ normal & $68 \%$ normal & & \\
\hline \multirow{2}{*}{ Cough } & $22(2.8)$ & $15(2.1)$ & $9(2.1)$ & \multirow{2}{*}{$<0.001$} & \multirow{2}{*}{$<0.02$} \\
\hline & $0 \%$ normal & $25 \%$ normal & $59 \%$ normal & & \\
\hline \multirow{2}{*}{ Hoarseness } & $19(2.1)$ & $14(1.1)$ & $5(1.0)$ & \multirow{2}{*}{$<0.001$} & \multirow{2}{*}{$<0.004$} \\
\hline & $14 \%$ normal & $30 \%$ normal & $77 \%$ normal & & \\
\hline \multirow{2}{*}{ Nasal leakage } & $13(3.3)$ & $7(1.9)$ & $5(1.4)$ & \multirow{2}{*}{$<0.004$} & \multirow{2}{*}{$<0.500$} \\
\hline & $41 \%$ normal & $65 \%$ normal & $77 \%$ normal & & \\
\hline
\end{tabular}

At late follow-up 21 (95\%) exhibited a normal MOT score. The mean MOT score for the whole group improved significantly $(0.5$, range $0-2.6, \mathrm{p}<0.003)$. Only nasal leakage did not improve significantly at this timepoint (Table 1 , Figures $4 \& 5$ ). c) VAS: At baseline, only one patient (5\%) reported a normal level. At end-of-training this had improved to 10 (45\%) and at long term follow-up this number had increased further to 15 (68\%) - (Figures $1 \& 2$, Table 4) .

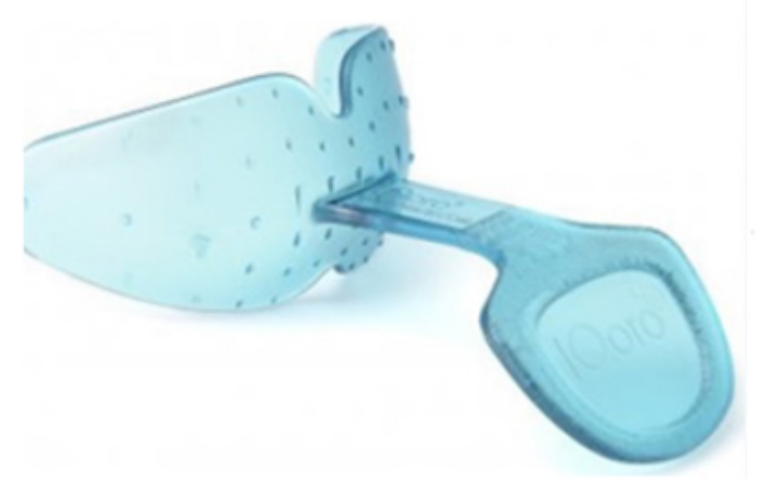

a) IQoro neuromuscular training device.

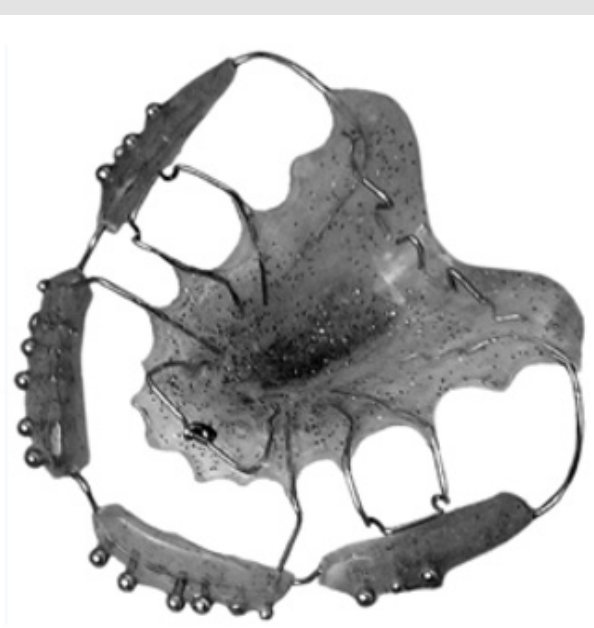

b) Palatal plate

Figure 1. 

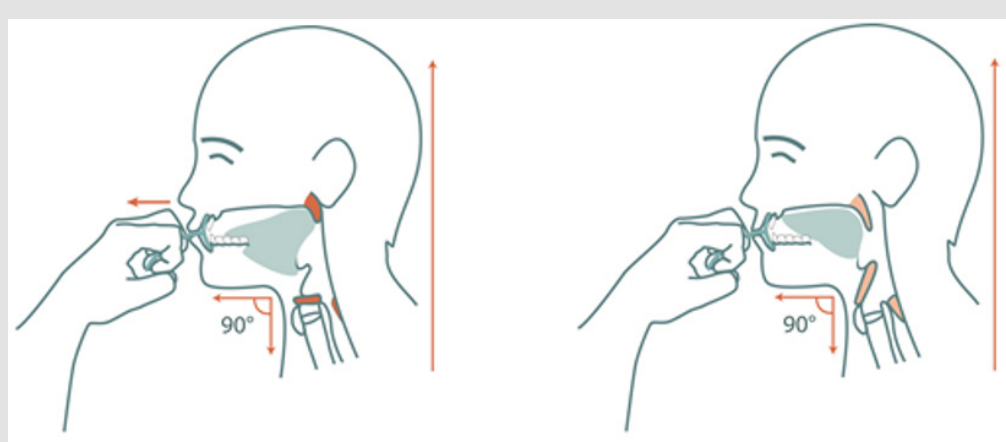

Figure 2: A) and B). Oral neuromuscular training with the IQoro® device.

A) The IQoro ${ }^{\circ}$ device is inserted pre-dentally behind closed lips. B) The patient presses the lips together firmly and pulls straight forward strongly for $5-10$ s and repeats the exercise three times with a $3 \mathrm{~s}$ rest between each. Training should be performed 3-times a day, ideally before mealtimes. Illustrations: courtesy Mary Hägg

\section{Correlation between Test Results}

At baseline, there was very high consistency between TWST, MOT and VAS results - all showing a Spearman correlation of over 90\% (Table $2 \& 3$ and Figures 3-5). At end-of-training the correlation between TWST and VAS remained high at $82 \%$, the other correlations reduced, but with the MOT / TWST remaining higher than the MOT / VAS (Tables 2-4 and Figures 4 \& 5). At long term follow-up, VAS correlated with a 73\% level to both TWST and MOT. The two objective tests, TWST and MOT recorded a lower correlation (Table 2 and Figure 5).

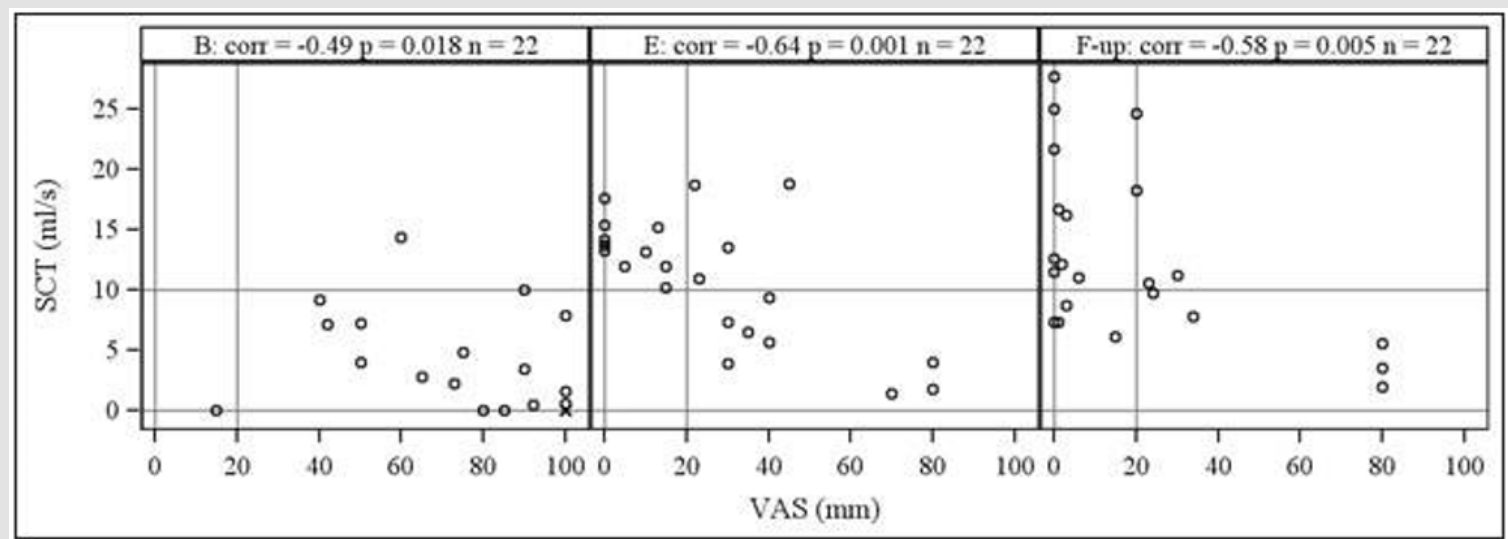

Figure 3: Timed Swallow Test (TWST) versus Visual Analogue Scale (VAS).

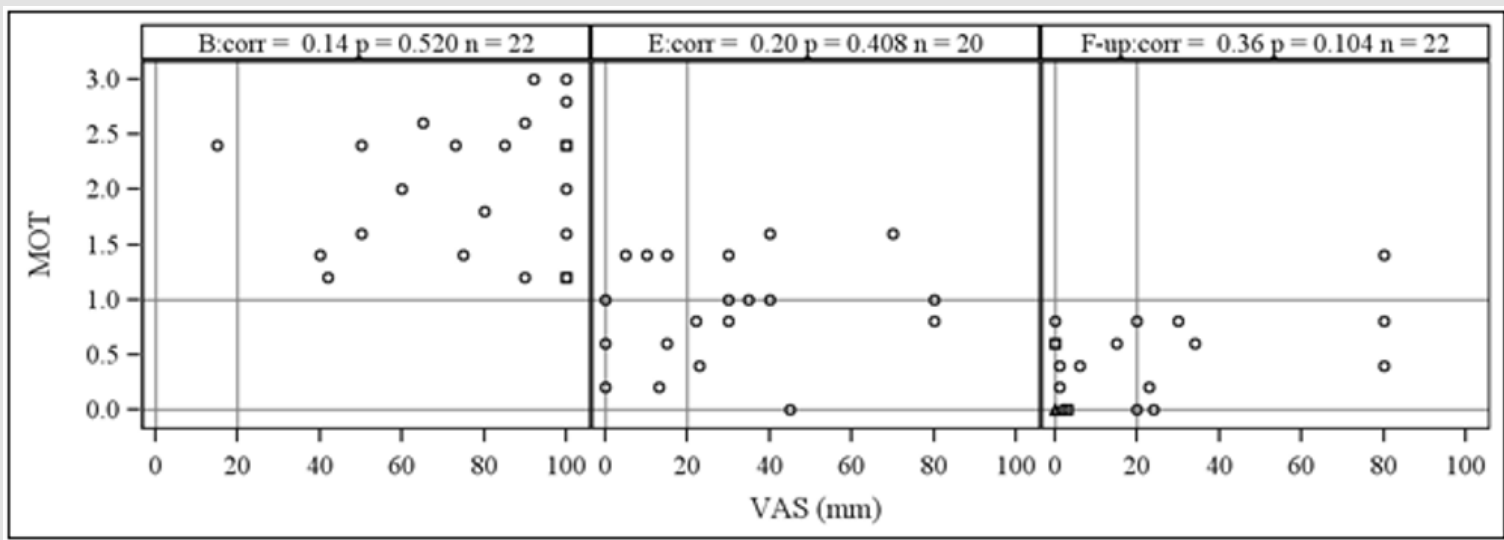

Figure 4: Meal observation test (MOT) versus visual analogue scale (VAS).

Table 2: Agreement in classification of patients as normal/pathological by visit.

\begin{tabular}{|c|c|c|c|}
\hline Agreement & Baseline & End & Follow-up \\
\hline TWST v. VAS & $91 \%(\mathrm{n}=22)$ & $82 \%(\mathrm{n}=22)$ & $73 \%(\mathrm{n}=22)$ \\
\hline MOT v. VAS & $96 \%(\mathrm{n}=22)$ & $40 \%(\mathrm{n}=20)$ & $73 \%(\mathrm{n}=22)$ \\
\hline MOT v. TWST & $91 \%(\mathrm{n}=22)$ & $60 \%(\mathrm{n}=20)$ & $64 \%(\mathrm{n}=22)$ \\
\hline
\end{tabular}




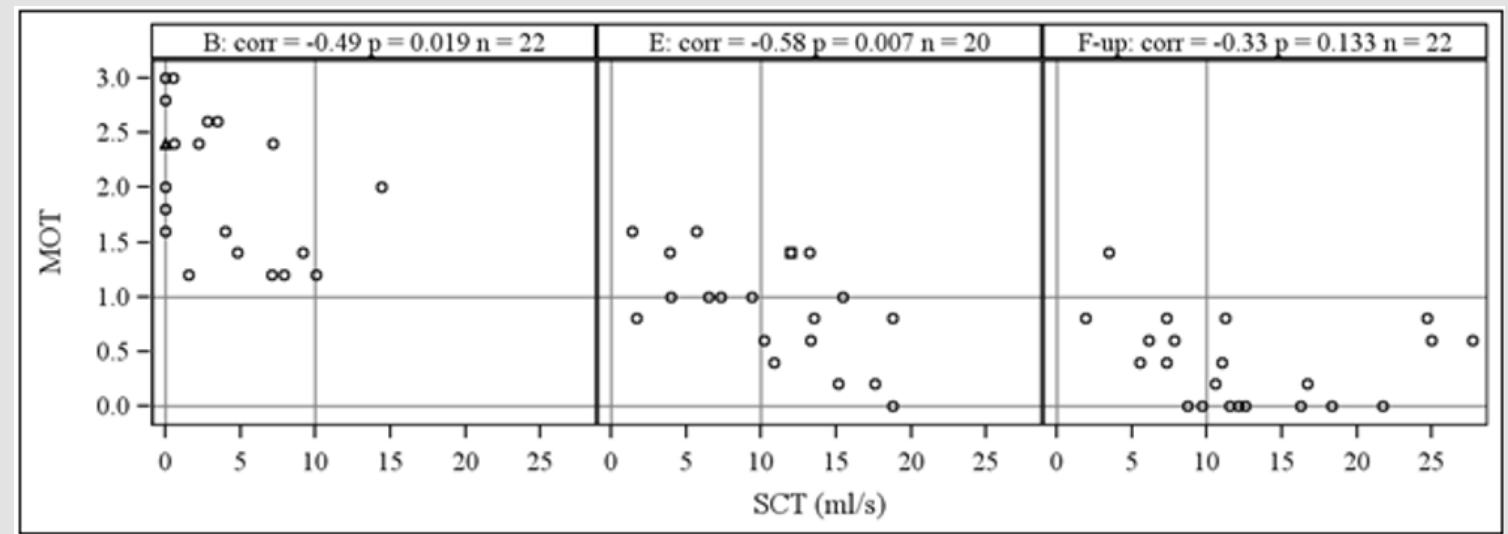

Figure 5: Meal Observation Test (MOT) versus Timed Water Swallow Test (TWST).

Table 3: Spearman correlation by time point.

\begin{tabular}{|c|c|c|c|}
\hline Correlation & Baseline & End & Follow-up \\
\hline TWST v. VAS & corr $=-0.49, \mathrm{p}=0.018 \mathrm{n}=22$ & $\operatorname{corr}=-0.64, \mathrm{p}=0.001 \mathrm{n}=22$ & $\operatorname{corr}=-0.58, \mathrm{p}=0.005 \quad \mathrm{n}=22$ \\
\hline MOT v. VAS & $\operatorname{corr}=0.14, \mathrm{p}=0.520 \mathrm{n}=22$ & $\operatorname{corr}=0.20, \mathrm{p}=0.408 \mathrm{n}=20 \quad \mathrm{corr}=0.36, \mathrm{p}=0.104 \quad \mathrm{n}=22$ \\
\hline MOT v. TWST & $\operatorname{corr}=-0.49, \mathrm{p}=0.019 \mathrm{n}=22$ & $\operatorname{corr}=-0.58 \mathrm{p}=0.007 \mathrm{n}=20 \quad \operatorname{corr}=-0.33, \mathrm{p}=0.133 \quad \mathrm{n}=22 \quad$ \\
\hline
\end{tabular}

Table 4: Change in TWST, MOT and VAS, $\mathrm{n}$ (median); min to max; p-value from Wilcoxon's test.

\begin{tabular}{|c|c|c|c|c|c|}
\hline Item & Baseline & End & Follow-up & $\begin{array}{c}\text { B-E } \\
\text { p-value }\end{array}$ & $\begin{array}{c}\text { E-FU } \\
\text { p-value }\end{array}$ \\
\hline TWST & $22(1.6) ; 0-14$ & $22(12.0) ; 1-19$ & $22(11.1) ; 2-28$ & $<.0001$ & 0.2842 \\
\hline MOT & $22(2.2) ; 1-3$ & $20(1.0) ; 0-2$ & $22(0.4) ; 0-1$ & $<.0001$ & 0.0028 \\
\hline VAS & $22(90.0) ; 15-100$ & $22(22.5) ; 0-80$ & $22(4.5) ; 0-80$ & $<.0001$ & 0.1020 \\
\hline
\end{tabular}

The statistical significance rating for these Spearman correlation figures was highest between TWST and VAS (Figure 3), and lowest between MOT and VAS which returned a non-significant score (Figure 4). In diagnosing OPD, all three methodologies distinguished similarly between pathological and normal swallowing abilities. Agreement between all three test methods was high at baseline, end-of-treatment and at long-term follow up. The weakest agreement was between MOT and VAS at the end-oftreatment time point.

The Spearman analysis showed significant correlation between TWST vs. VAS, and MOT vs. TWST at all time points, except MOT vs. TWST at long-term follow up. In this correlation analysis MOT v VAS did not show significant agreement.

\section{Discussion}

\section{Tests Overview}

Two of these tests - TWST and MOT - are conducted by independent observers and can be thought of as more or less objective. The VAS test asks the patient's own perception of how the swallowing dysfunction exhibits itself in the home setting.

\section{TWST}

The TWST is well-validated and used extensively in many countries with high inter-, intra- and test retest reliability. Correlation with this test would give credence to both MOT and VAS as very useful tools in assessing OPD and its treatment. It is simple, easy and cheap to administer and its outcomes have been shown to also have a strong correlation to misdirected swallowing. Wu et. al. reported in 2004 that 47 of 49 patients with swallowing rates of less than $10 \mathrm{ml} / \mathrm{s}$ also showed video radiographic signs of swallowing dysfunction [11]. A modified $30 \mathrm{ml}$ water swallowing test has been regarded as a useful single-task screening tool for detecting aspiration [12]. However, this test does not take other OPD factors into account. In a review, Bours et al. [13] concluded that a water test combined with pulse oximetry using coughing, choking, and voice alterations as endpoints is the best method for screening patients with neurological disorders for dysphagia. However, these endpoints only reflect factors of importance for aspiration and will not detect other OPD components.

\section{MOT}

The MOT is a real-life test of eating ability - which is more than just swallowing function. It includes both solid and liquid intake and provides observers with a variety of measurable variables. It also takes more time than the VAS or TWST tests and fatigue, lack of teeth, environmental distractions and other factors may be at play too. The MOT can provide information about aspiration, intraoral bolus control, drooling, and leakage through the nose. 'Cough at meals' and 'hoarseness after a meal are indicators of aspiration, which can be due to pharyngeal or hypopharyngeal sensory loss or a muscular inability to close the laryngeal airway. Tongue paresis would result in a long oral transit time and an inability to control oropharyngeal bolus transit and can cause aspiration. Leakage through the nose may indicate soft palatal paresis, and drooling 
can be a sign of facial paresis, an inability to close the mouth, or sensory loss. These disabilities are some of the characteristics of OPD. If aspiration occurs without coughing, the vocal cords cannot provide a clear voice, resulting in hoarseness after a meal. Silent aspiration may occur during a meal when hoarseness is present even without coughing. In our study of patients with more or less chronic OPD, there were apparently no silent aspirators before therapy, because all patients with hoarseness were coughing during the meal. In 2005, Lambert et al. [2] showed the validity of a meal test in neurological patients with eating disabilities to predict short survival time.

\section{VAS}

Self-reporting tests like VAS are powerful, but one should guard against the fact that the patient's state of mind can be different at baseline, end-of-treatment and long-term follow-up. In some ways it is 'not the same person' answering the VAS at each timepoint. The level of understanding of the gravity of his condition, expectations of his future life, relief at having survived a stroke, etc. can all affect his base position when reporting the effect of his swallowing dysfunction on his daily life. Patients tend to adapt their eating and drinking activities to reflect their new ability levels; again, providing a moving basepoint from which to judge their current condition. The effect of these factors is not known, and it could be that they might cause an individual to report an unchanged condition with a higher or lower VAS score at different times.

\section{VF and FEES}

Tests not used in this study include Videofluoroscopy (VF) and Fiber optic endoscopic evaluation (FEES). VF has been regarded as the diagnostic method of choice for silent aspiration [12,14]. The method is expensive and requires specialized staff and techniques [14]. (FEES) [15,16] is another suitable method for detecting aspiration, even silent aspiration, and has been used in acute stroke to predict the necessity of endotracheal intubation.

\section{Patient Group}

It should be noted that the majority of patients were not recent stroke victims, the proposed early intervention group was depleted by mortality or second stroke before inclusion. The included patients will mostly not have been in post-stroke shock when they reported their baseline VAS scores. Note also that this cohort had severe problems, the majority recording pathological scores on all tests. Despite this, improvements were significant and widespread and were reliably detected by all three test methodologies.

\section{Conclusion}

TWST, MOT and VAS are all reliable tools for the clinician who wishes to diagnose OPD, and to track the progress of rehabilitation treatment. All have the advantage of being simple to carry out in any environment and by any healthcare professional.

It seems that TWST has the widest degree of scientific evidence, and that both MOT and VAS give similar levels of certainty in diagnosing OPD. The lack of correlation of VAS with the other test methodologies suggests that caution is needed when deploying this method alone in patient evaluation; this may because of the hypothesised phenomenon of the patient's observation baseline changing over time. This study demonstrates powerful alternatives to the clinician when preferred, or when more specialist VF or FEES resources are not readily available.

\section{Acknowledgement}

The authors wish to thank bio-statistician Lisa Wernroth and clinical data manager Manuela Zamfir of the Uppsala Clinical Research Center for constructing the database and analyzing the data.

\section{References}

1. Lakshminarayan K, Tsai AW, Tong X, Vazquez G, Peacock JM, et al. (2010) Utility of dysphagia screening results in predicting poststroke pneumonia. Stroke 41(12): 2849-2854.

2. Lambert HC, Abrahamowicz M, Groher M, Wood-Dauphinee S, Gisel EG (2005) The McGill ingest skills assessment predicts time to death in an elderly population with neurogenic dysphagia: preliminary evidence. Dysphagia 20(2): 123-132.

3. Hägglund P, Fält A, Hägg M, Wester P, Levring Jäghagen E (2018) Swallowing dysfunction as risk factor for undernutrition in older people admitted to Swedish short-term care: a cross-sectional study. Aging Clin Exp Res 31(1): 85-94.

4. Lugger KE (1994) Dysphagia in the elderly stroke patient. J Neurosci Nurs 26(2): 78-84.

5. Guigoz Y (2006) The Mini Nutritional Assessment (MNA) review of the literature - What does it tell us? J Nutr Health Aging 10(6): 466-485.

6. Hägglund P, Hägg M, Wester P, Levring Jäghagen E (2019) Effects of neuromuscular treatment with an oral training device on swallowing dysfunction among older people in short-term care - a cluster randomized, controlled trial. Age and Ageing 48(4): 533-540.

7. Hägg M, Anniko M (2008) Lip muscle training in stroke patients with dysphagia. Acta Oto-Laryngologica 128: 1027-1033.

8. Nathadwarawala KM, Nicklin J, Wiles CM (1992) A timed test of swallowing capacity for neurological patients. J Neurol Neurosurg Psychiatry 55: 822-825.

9. Kalyanee M, Nathadwarawala KM, McGroary A, Wiles CM (1994) Swallowing in neurological outpatients. Use of a timed test. Dysphagia $9(2): 120-129$.

10. Hägg M, Larsson B (2004) Effects of motor and sensory stimulation in stroke patients with long-lasting dysphagia. Dysphagia 19(4): 219-230.

11. Wu MC, Chang YC, Wang TG (2004) Evaluating swallowing dysfunction using a 100-ml water swallowing test. Dysphagia 19(1): 43-47.

12. Nishiwaki K, Tsuji T, Liu M, Hase K, Tanaka N, et al. (2005) Identification of a simple screening tool for dysphagia in patients with stroke using factor analysis of multiple dysphagia variables. J Rehabil Med 37: 247251.

13. Bours GJ, Speyer R, Lemmens J, Limburg M, de Wit R (2009) Bedside screening tests vs. ideofluoroscopy or fiberoptic endoscopic evaluation of swallowing to detect dysphagia in patients with neurological disorders: systematic review. J Adv Nurs 65: 477-493.

14. Logemann J A (1988) Evaluation and treatment of swallowing disorders. ( $2^{\text {nd }}$ edn.). Austin, TX: Pro, ed, pp. 406.

15. Dziewas R, Warnecke T, Olenberg S, Teismann I, Zimmermann J, et al. (2008) Towards a basic endoscopic assessment of swallowing in acute stroke -development and evaluation of a simple dysphagia score. Cerebrovasc Dis 26: 41-47.

16. Warnecke T, Ritter MA, Kroger B, Oelenberg S, Teismann I, et al. (2009) Fiberoptic endoscopic Dysphagia severity scale predicts outcome after acute stroke. Cerebrovasc Dis 28(3): 283-289. 


\section{ISSN: 2574-1241}

DOI: 10.26717/BJSTR.2019.21.003623

Mary Hägg. Biomed J Sci \& Tech Res

(C) (i) This work is licensed under Creative

Submission Link: https://biomedres.us/submit-manuscript.php

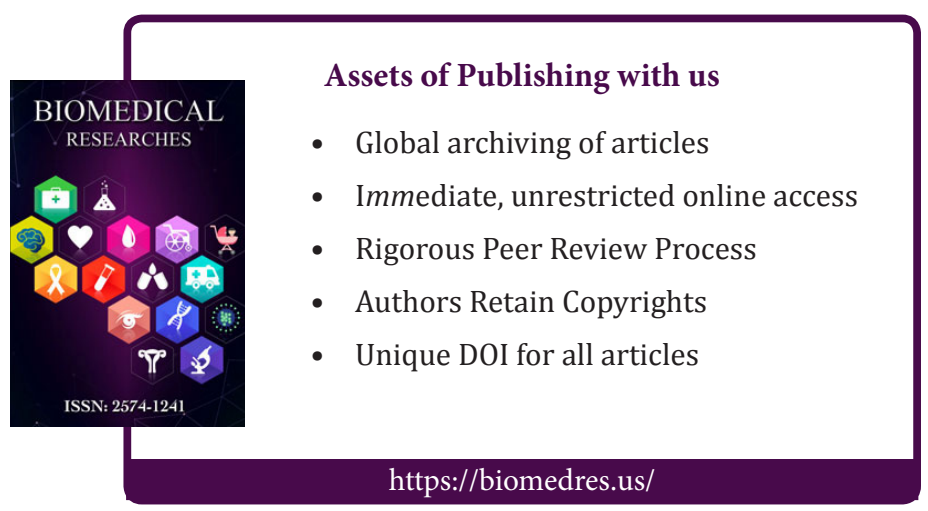

